\title{
Effects of silk solution against laminectomy-induced dural adhesion formation and inflammation in a rat model
}

\author{
${ }^{*}$ Yong-Jun Cho, MD, ${ }^{1}$ Chi Hern Lee, MA, ${ }^{2}$ Dae Won Kim, PhD, ${ }^{3}$ Ki-Yeon Yoo, PhD, ${ }^{4}$ \\ Won Sik Eum, PhD, ${ }^{2}$ Min Jea Shin, PhD, ${ }^{2}$ Hyo Sang Jo, PhD, ${ }^{2}$ Jinseu Park, PhD, ${ }^{2}$ \\ Kyu Hyung Han, PhD, ${ }^{2}$ Keun Wook Lee, PhD, ${ }^{2}$ and Soo Young Choi, PhD ${ }^{2}$
}

\begin{abstract}
1Department of Neurosurgery, Hallym University Medical Center, Chuncheon; ${ }^{2}$ Department of Biomedical Science and Research Institute of Bioscience and Biotechnology, Hallym University, Chuncheon; ${ }^{3}$ Department of Biochemistry and Molecular Biology, Research Institute of Oral Sciences, College of Dentistry, Gangneung-Wonju National University, Gangneung; and ${ }^{4}$ Department of Oral Anatomy, College of Dentistry and Research Institute of Oral Biology, Gangneung-Wonju National University, Gangneung, Korea
\end{abstract}

OBJECTIVE Adhesion formation is one of the most common complications following laminectomy. The efficiency of antiadhesive agents is required to prevent postsurgical adhesion. Therefore, the authors investigated the effects of silk solution against laminectomy-induced dural adhesion formation in a rat model.

METHODS The authors prepared the silk solution from silkworms. Laminectomies were performed between L3 and L5 in all rats. MediShield was used as a control. The effects of silk solution against laminectomy-induced dural adhesion formation and inflammation were confirmed by histological examination and Western blot analysis.

RESULTS Laminectomy-induced dural adhesion formation was drastically reduced in silk solution-treated rats (Grade 2) compared to vehicle-treated rats (Grade 3). Dural adhesion formation was significantly reduced in rats that received treatment with silk solution or MediShield, which is a known antiadhesion drug. In addition, combined treatment with silk solution and MediShield showed greater reductions in inflammation compared to the silk solution-or MediShield-treated rats $(p>0.05)$.

CONCLUSIONS In this study, the authors demonstrated that silk solution effectively reduced postlaminectomy dural adhesion formation in rats. Although further studies on the long-term effects are necessary to validate these results, silk solution may potentially serve as an antiadhesion agent in neurological procedures.

https://thejns.org/doi/abs/10.3171/2018.4.SPINE171164

KEYWORDS antiadhesion; laminectomy; rat; silk solution; inflammation

$\mathrm{S}$ EVERAL studies have demonstrated that the lesion after a spinal cord injury expands to secondary degenerative processes, including vascular disturbances, metabolic failure, ionic dysregulation, excitotoxicity, free radical formation, cytokine release, and inflammation. Therefore, treatment strategies for human spinal cord injury have been designed to target several aspects of secondary injury. $8,11,36$

Laminectomy is a commonly performed spinal surgery to decompress the spinal cord and nerve roots. Unfortunately, $8 \%-40 \%$ of patients suffer from this syndrome of spinal cord/nerve root compression, and adhesion is known to occur as a result of surgery in up to $34 \% \%^{3,13,21,27}$ of patients. Although various methods have been developed to prevent postsurgical adhesion formation, no effective treatment exists. $5,7,14,32,40,44$ Thus, postsurgical adhesion formation has emerged as one of the major problems in these surgical procedures.

Although various factors are highly associated with epidural fibrosis, epidural fibrosis is known to play a key role in adhesion formation, and inflammation is a critical factor causing epidermal fibrosis and adhesion formation..$^{20,37,45}$ Several studies using rat laminectomy models have demonstrated that treatment using various agents

ABBREVIATIONS COX-2 = cyclooxygenase-2; IL = interleukin; iNOS = inducible nitric oxide synthase; MMP = matrix metalloproteinase. SUBMITTED October 20, 2017. ACCEPTED April 2, 2018.

INCLUDE WHEN CITING Published online August 24, 2018; DOI: 10.3171/2018.4.SPINE171164.

${ }^{*}$ Y.J.C., C.H.L., and D.W.K. contributed equally to this work. 
may inhibit postsurgical adhesion formation by reducing inflammation. $5,6,37$

Silk fibroin from Bombyx mori silkworms has been used for a variety of biomedical purposes, including as surgical sutures, because of its excellent biological and mechanical properties, lack of cytotoxicity, and low host inflammatory reaction. ${ }^{15,22-24}$ Rockwood et al. demonstrated that silk fibroin has been successfully used in various material forms, including films, sponges, hydrogels, and microparticles. ${ }^{30}$ In a previous study, we demonstrated that transparent, artificial dura mater from silk fibroin may have clinical applications in neurosurgical procedures. ${ }^{17}$ However, the effects of silk solution against laminectomy-induced adhesion formation are unclear. Thus, we investigated the effects of silk solution against laminectomy-induced adhesion formation and inflammation in a rat model.

\section{Methods}

\section{Preparation of the Silk Solution}

Silk fibroin from the cocoons of Bomvyx mori silkworms was obtained from the Rural Development Administration in Jeonju, Korea. Briefly, cocoons were boiled with $0.02 \mathrm{M} \mathrm{Na}_{2} \mathrm{CO}_{3}$ for 1 hour, washed 5 times with distilled water to remove sericin proteins, and then dried using clean convection air for 12 hours. The dried fibroin fibers were dissolved using 9.3 $\mathrm{M} \mathrm{LiBr}$ solution for 4 hours at $60^{\circ} \mathrm{C}$ to disrupt hydrogen bonding between the fibroin protein chains. Then, the $\mathrm{LiBr}$ was removed from the silk solution by dialysis (3500-Da membrane) against pure water over 48 hours. ${ }^{18}$ The final silk solution concentration was about $8 \%$ weight/volume. The silk solution was mixed with glycerol in a ratio of 8:2.

\section{Experimental Animals and Surgical Procedure}

This study used Sprague-Dawley rats obtained from the Experimental Animal Center at Hallym University. The animals were housed at a constant temperature $\left(23^{\circ} \mathrm{C}\right)$ and relative humidity $(60 \%)$ with a fixed 12 -hour light/dark cycle and free access to food and water. All procedures regarding animals and their care conform to the Guide for the Care and Use of Laboratory Animals of the National Veterinary Research \& Quarantine Service of Korea and were approved by the Hallym Medical Center Institutional Animal Care and Use Committee.

Rats ( $\mathrm{n}=5$ /group) were divided into 5 groups as follows: 1) a sham normal control group, 2) a laminectomyinduced adhesion vehicle group, 3 ) single treatment with silk $(8 \% / 1 \mathrm{ml})$ in a laminectomy-induced adhesion group, 4) single treatment with MediShield (antiadhesive gel [1 ml]; Medtronic International Trading SARL) in a laminectomy-induced adhesion group, and 5) single treatment with silk $(8 \% / 0.5 \mathrm{ml})$ and MediShield $(0.5 \mathrm{ml})$ in a laminectomy-induced adhesion group.

To determine the effects of silk solution against laminectomy-induced adhesion formation in the rat animal model, rats were anesthetized by a single intraperitoneal injection of $0.2 \mathrm{ml}$ of tiletamine/zolazepam $\mathrm{HCl}$ (Zoletil) and xylazine $\mathrm{HCl}$ (Rompun, Bayer). The lower back of each rat was shaved and sterilized with povidone. An L2-5 skin incision was performed, followed by lumbosacral fas- ciotomy and subperiosteal paraspinous muscle dissection, exposing the L3-5 laminae. Finally, the dura mater was fully exposed after total L3-5 laminectomy, flavectomy, and epidural fat tissue removal. Hemostasis was achieved with cotton pads. The rats were then randomly allocated into 5 groups of 5 rats each as described above. After silk solution $(8 \% / 1 \mathrm{ml})$ and/or MediShield $(1 \mathrm{ml})$ was applied as appropriate at L3-5, the layers were closed anatomically with the same suture material (Prolene polypropylene sutures, Ethicon Endosurgery, Inc.) in all rats. No complications or adverse effects arose from etanercept use in the animals. Four weeks postoperatively, the animals were sacrificed, and their T10-L5 vertebral columns were removed en bloc and then placed in 10\% buffered formalin. ${ }^{38}$

Fixed specimens were cut into 3-mm thickness using a dental disk completely. Decalcification was carried out in a Decalcifying Solution-Lite (Sigma) at room temperature for 8 hours and checked for decalcification every 2 hours. The endpoint of the decalcification process was assessed with a surgical blade.

\section{Immunohistochemistry}

For histological analysis, rats were sacrificed 4 weeks postoperatively, and tissue specimens were extracted. Then the tissue specimens were cryoprotected, frozen, and sectioned $(10 \mu \mathrm{m})$. Masson's trichrome immunostaining was performed to evaluate the surrounding connective tissues. Briefly, the specimens were decalcified with ethylenediaminetetraacetic acid, dehydrated, and embedded in paraffin. Then, the sections were immunostained with Masson's trichrome. The epidural adhesion grading scheme devised by He et al. (Grade 0: no adhesion, Grade 1: slight adhesion, Grade 2: moderate adhesion, and Grade 3: severe adhesion) was used to grade adhesion fibrosis in each specimen. ${ }^{12}$

Matrix metalloproteinases 2 and 9 (MMP-2 and MMP-9) and ED1 expression levels in specimens were determined by immunostaining. The sections were incubated with indicated specific antibodies and appropriate secondary antibodies. Then, images were obtained using the CCD camera of an Axiophot light microscope (Carl Zeiss). Expression levels were determined using an image analysis system equipped with a computer-based CCD camera (Optimas 6.5 software, CyberMetrics).

\section{Western Blot Analysis}

We examined the effects of silk solution against inflammatory response in a dural adhesion rat animal model using Western blot analysis. The tissue specimens $(1 \times 1-\mathrm{cm}$ region of adhesion) were homogenized, lysed, and centrifuged. Then, the same protein samples were resolved by $18 \%$ sodium dodecyl sulfate-polyacrylamide gel electrophoresis and transferred into a nitrocellulose membrane. The membranes were blocked with 5\% nonfat dry milk and washed with TBST buffer $(25 \mathrm{mM}$ Tris- $\mathrm{HCl}$, $140 \mathrm{mM} \mathrm{NaCl}, 0.1 \%$ Tween 20, pH 7.5). Subsequently, the membranes were immunoblotted with primary antibodies (MMP-2, MMP-9, ED1, CD34, cyclooxygenase [COX]-2, inducible nitric oxide synthase [iNOS], interleukin [IL]- 
$1 \beta$, IL-6, IL-12, and beta-actin were obtained from Cell Signaling Technology or Abcam) for 1 hour and with the appropriate secondary antibodies for 1 hour. The protein bands were detected using ECL solution (Amersham) according to the manufacturer's instructions.

\section{Statistical Analysis}

Data are expressed as the mean \pm SEM. The data were analyzed using one-way ANOVA to determine statistical significance. Bonferroni's test was used for post hoc comparisons. A value of $p<0.05$ was considered statistically significant.

\section{Results \\ Effect of Silk Solution Against Adhesion After Laminectomy}

Postsurgical adhesion formation has emerged as one of the major problems following laminectomy. Thus, we prepared silk solution and examined its effect on postlaminectomy dural adhesion formation in a rat animal model. As shown in Fig. 1, dural adhesion formation was markedly increased in the vehicle group (Grade 3), whereas dural adhesion formation levels were reduced in the silk solution (Grade 2) and MediShield (Grade 2) treatment groups compared to the vehicle treatment group. However, combined treatment with silk solution and MediShield more efficiently reduced dural adhesion formation levels (Grade 1).

\section{Effect of Silk Solution Against Laminectomy-Induced Inflammation}

Since inflammation is one of the most important factors causing dural adhesion formation, the effects of silk solution on the inhibition of the inflammatory response mediator's expression levels were investigated under the same experimental conditions. As shown in Figs. 2 and 3, MMP-2, MMP-9, and ED1 expression levels were significantly increased in the vehicle-treated group compared to the normal sham group. However, increased MMP-2, MMP-9, and ED1 expression levels were markedly reduced in specimens treated with silk solution $(p>0.05)$ or MediShield ( $p>0.05)$. Furthermore, combined treatment with silk solution and MediShield more efficiently reduced MMP-2, MMP-9, and ED1 expression levels ( $p$ > 0.01).

Furthermore, we determined the effects of silk solution against laminectomy-induced CD34, COX-2, iNOS, and cytokine expression levels. As shown in Fig. 4, laminectomy-induced CD34, COX-2, and iNOS expression levels were significantly reduced in both the silk solution- and MediShield-treated groups ( $p>0.01)$. Cytokine expression levels including IL-1 $\beta$, IL-6, and IL-12 were also markedly reduced in the silk solution- and MediShield-treated groups ( $p>0.05)$. In addition, combined treatment with silk solution and MediShield more efficiently reduced CD34, COX-2, iNOS, and cytokine expression levels $(\mathrm{p}>0.01)$.

\section{Discussion}

Dural adhesion formation is one of the most common complications following spinal surgery. Adhesion for- mation is known as an inevitable consequence of spinal laminectomy. Therefore, many patients suffer persistent pain, paresthesia, and motor weakness as a result of spinal injury. ${ }^{29}$ Also, scar formation is a key problem affecting $5 \%-30 \%$ of those undergoing spinal injury. ${ }^{6,29}$ Though several studies have demonstrated that various agents, including antiinflammatory drugs and antiadhesion gels, prevent postlaminectomy adhesion or scar formation, ${ }^{6,11} \mathrm{a}$ more efficient and effective treatment has not been found. Since adhesion and scar formation have emerged as one of the major problems in neurosurgical procedures, some researchers have suggested that the prevention of adhesion or scar formation may be the key goals in spinal injury. ${ }^{4}$

Many studies have shown that silk-based biomedical materials used in various forms have biocompatibility and nontoxic properties. ${ }^{15,22-24,30}$ Recently, Wang et al. demonstrated that silk-polyethylene glycol (PEG) hydrogels prevent adhesion and scar formation in a postlaminectomy rabbit model, suggesting that silk-PEG hydrogels may be responsible for effective adhesion prevention. ${ }^{39}$ We also demonstrated that silk-based transparent artificial dura mater may be used in neurosurgical procedures without toxic effects. ${ }^{17}$ Therefore, we examined the effects of the topical application of silk solution against laminectomyinduced dural adhesion formation and inflammation in a rat model. We designed an experiment to determine whether silk solution prevents postlaminectomy dural adhesion formation. As shown in Fig. 1, silk solution prevented dural adhesion formation levels in a fashion similar to treatment with MediShield. Since analysis of decalcification is limited by histological methods, the Decalcifying Solution-Lite used in this study is commercially available for general histological and immunohistochemical staining. In order to prevent antigenicity of decalcifying solution, we tried to shorten the decalcification time by minimizing the thickness of the tissue.

Several studies have shown that MediShield reduces dural adhesion formation and improves the symptoms of postlaminectomy syndrome. ${ }^{19,31,35}$ Our results are in agreement with these studies, indicating that silk solution has a positive effect on the prevention of postlaminectomy dural adhesion formation.

It is well known that inflammation is one of the key factors in dural adhesion formation and further that dural adhesion formation can be prevented by postlaminectomy inhibition of inflammation, and factors suggest that prevention of inflammation may be an effective strategy to reduce postlaminectomy dural adhesion or scar formation. ${ }^{6,20,37,45}$ We have shown that the application of silk solution significantly reduced expression levels of COX-2 and iNOS, which are involved in inflammatory response, in a laminectomy-induced adhesion animal rat model. ${ }^{17}$

MMPs are a family of zinc-dependent endopeptidases with various functions, including protein degradation in the extracellular matrix, cell migration, tissue damage and repair, and inflammation in pathological processes. ${ }^{25,42}$ Several studies have shown that MMP-2 and MMP-9 protein expression is involved in astrocyte migration, inflammation, and formation of a glial scar tissue in spinal cord injuries, suggesting that the inhibition of MMP-2 and MMP-9 may be a promising therapeutic target to reduce 


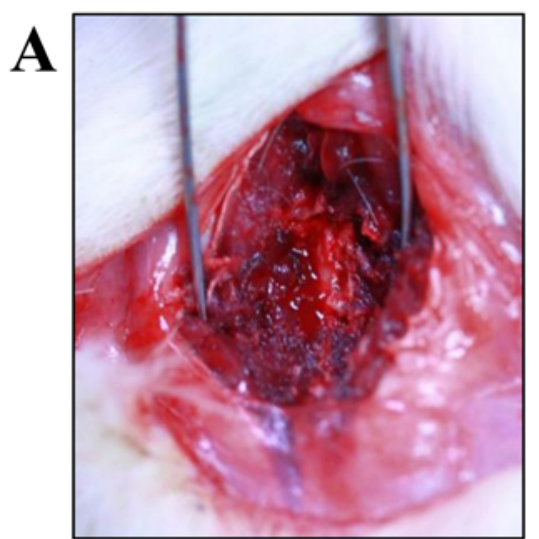

Dura exposed
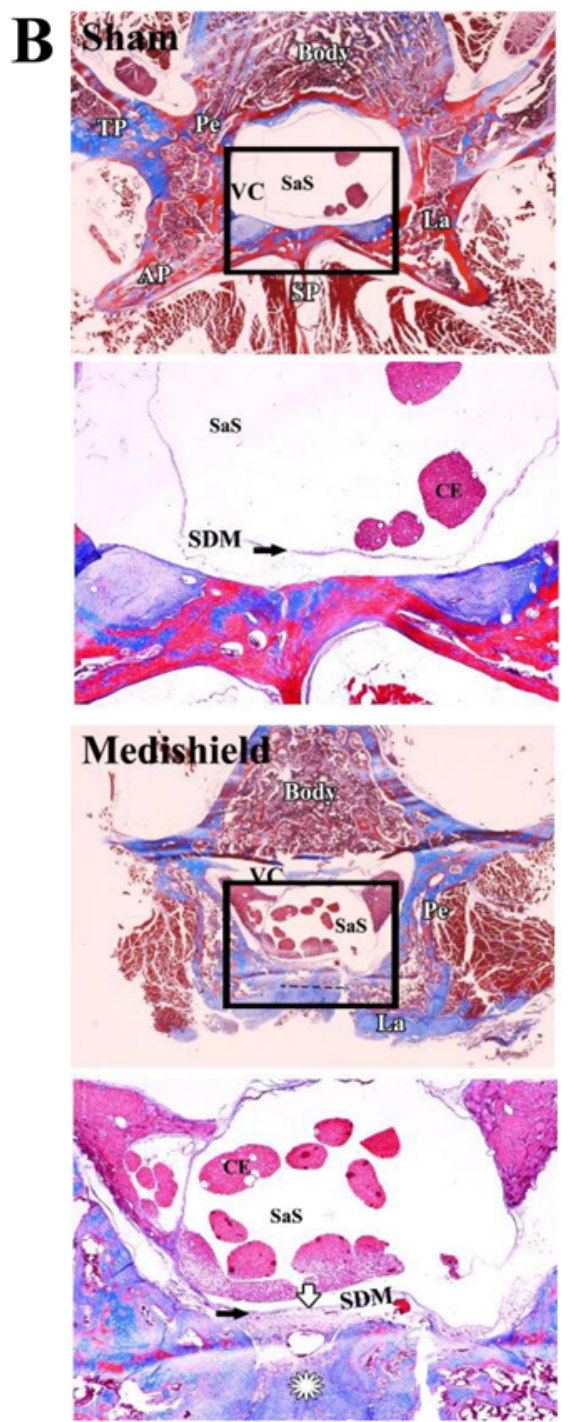

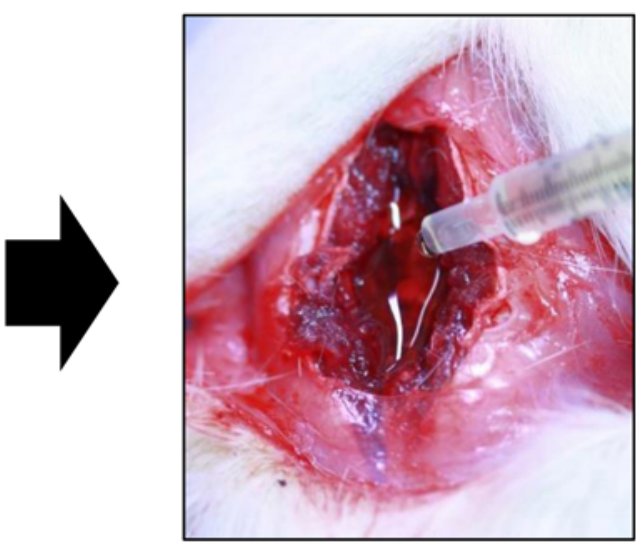

Covered by Silk+Medishield
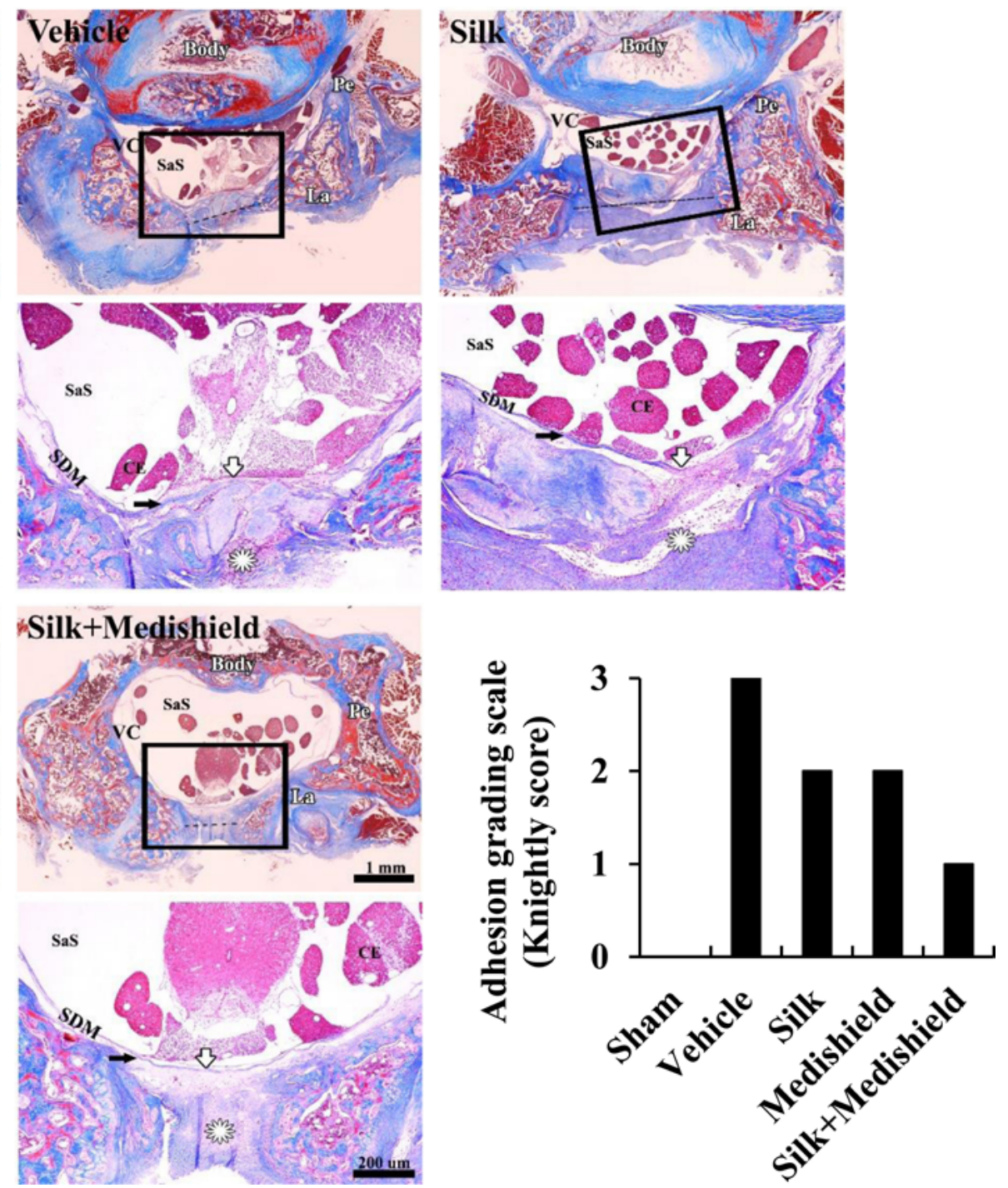

FIG. 1. Effects of silk solution against postlaminectomy rat dural adhesion formation. A: Photographs showing the dura exposed and the application of silk solution or MediShield in the rats. B: Representative images of dural adhesion formation in the sham control and vehicle-, silk solution-, MediShield-, and silk+MediShield-treated groups. Black arrows indicate the spinal dura mater; white arrows indicate dural adhesion; and asterisks indicate inflammation. Masson's trichrome staining. AP = articular process; CE = cauda equina; La = lamina; $\mathrm{Pe}$ = pedicle; $\mathrm{SaS}$ = subarachnoid space; SDM = spinal dura matter; $\mathrm{SP}=$ spinal cord; TP = transverse process; VC = vertebral cavity. Graph shows histological assessment scores: 0 points are in Grade 0, $\leq 1$ point is in Grade 1, $\leq 2$ points are in Grade 2, and $\leq 3$ points are in Grade 3. High-magnification images are shown in bottom panels. Scale bar $=1 \mathrm{~mm}$ (upper) and $200 \mu \mathrm{m}$ (lower). 

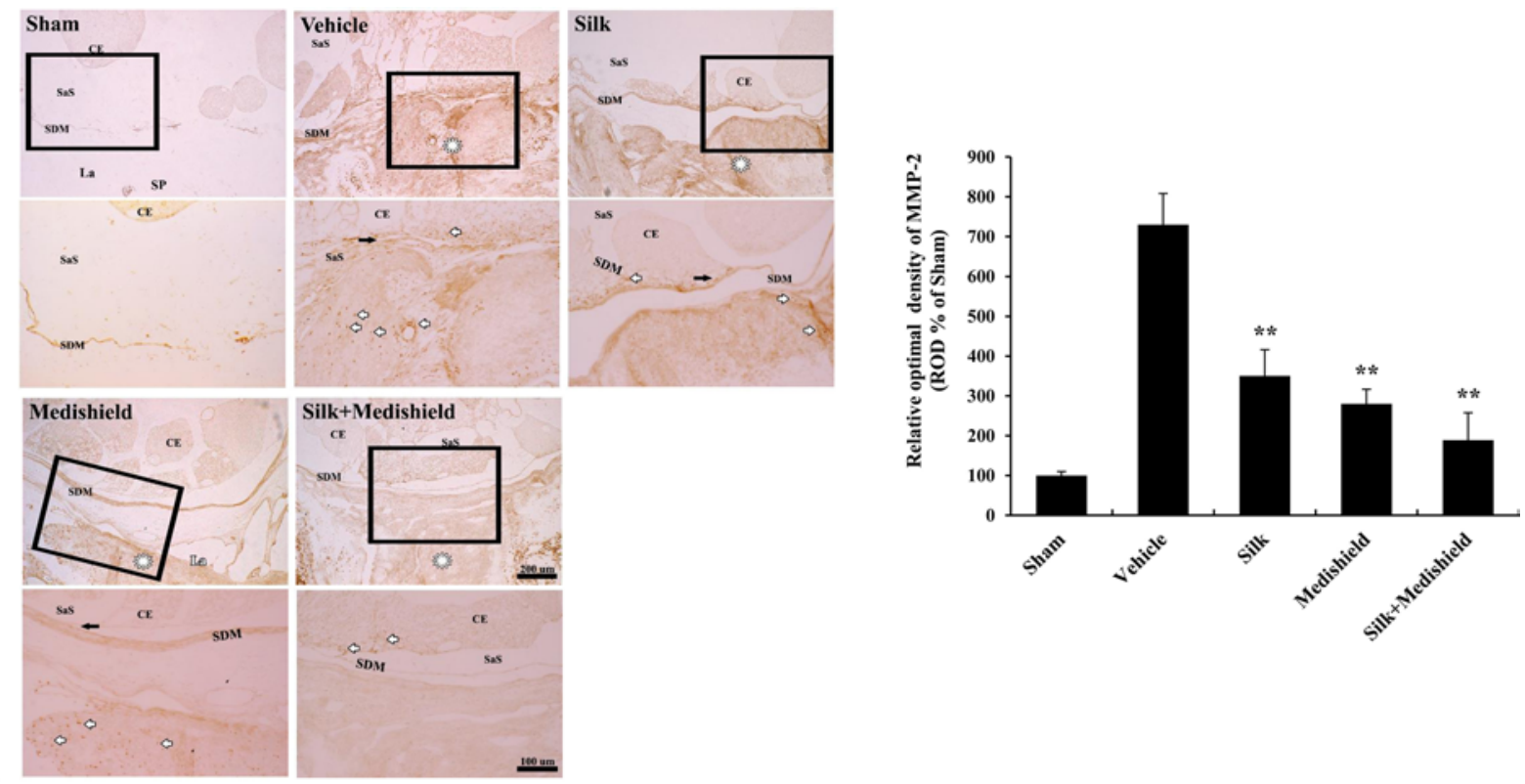

A
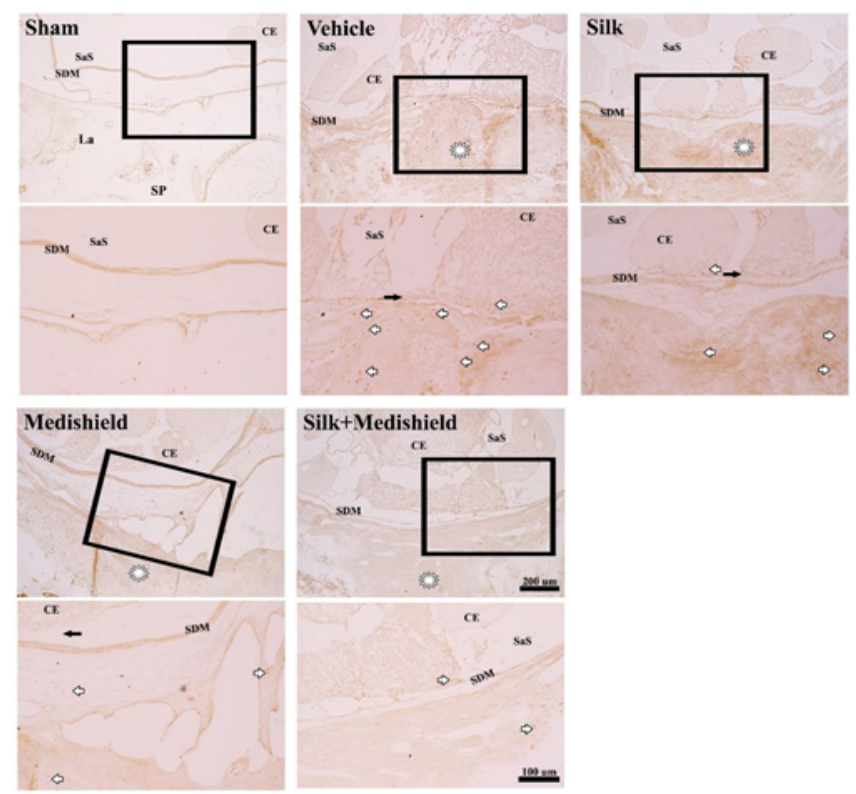

Silk+Medishield
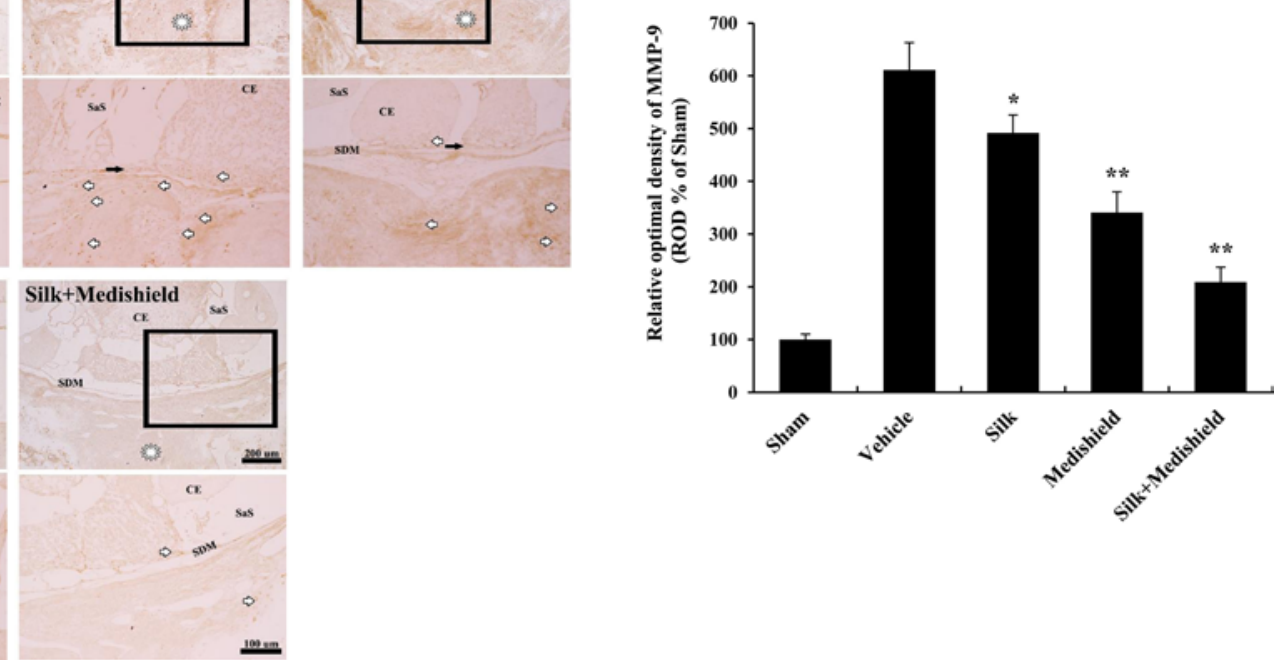

B
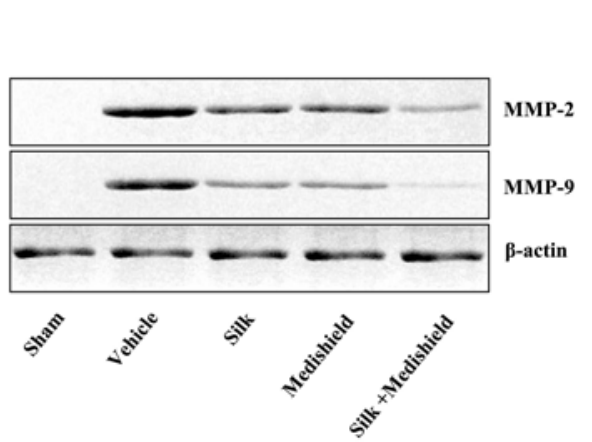

C

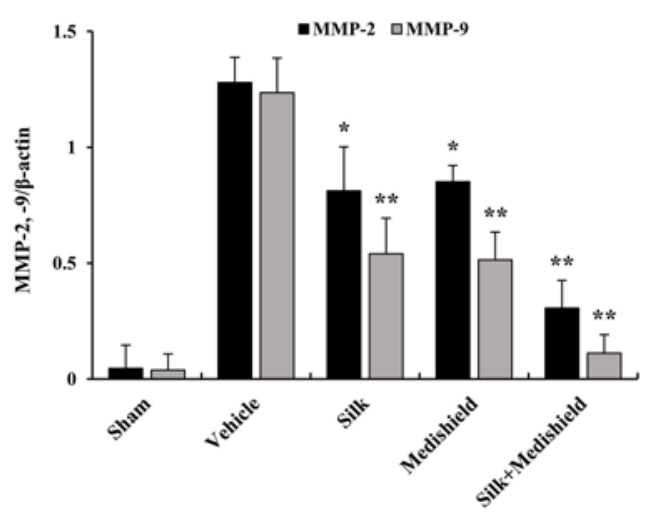

FIG. 2. Inhibitory effects of silk solution against MMP-2 and MMP-9 expression in the rat dural adhesion model. Histological and Western blot analysis of silk solution treatment of dural adhesion. Photomicrographs: MMP-2 (A) and MMP-9 (B) staining. Western blot analysis (C). Black arrows indicate the epidural adhesions; white arrows indicate the MMP-2 and MMP-9 immunoreactive cells. ROD = relative optimal density. High-magnification images are shown in lower panels. Scale bar $=200 \mu \mathrm{m}$ (upper) and $100 \mu \mathrm{m}$ (lower). The difference between the vehicle group and the silk solution-treated treated group was statistically significant (graphs: ${ }^{*} p<0.05$ and ${ }^{* *} p<0.01$ ). 

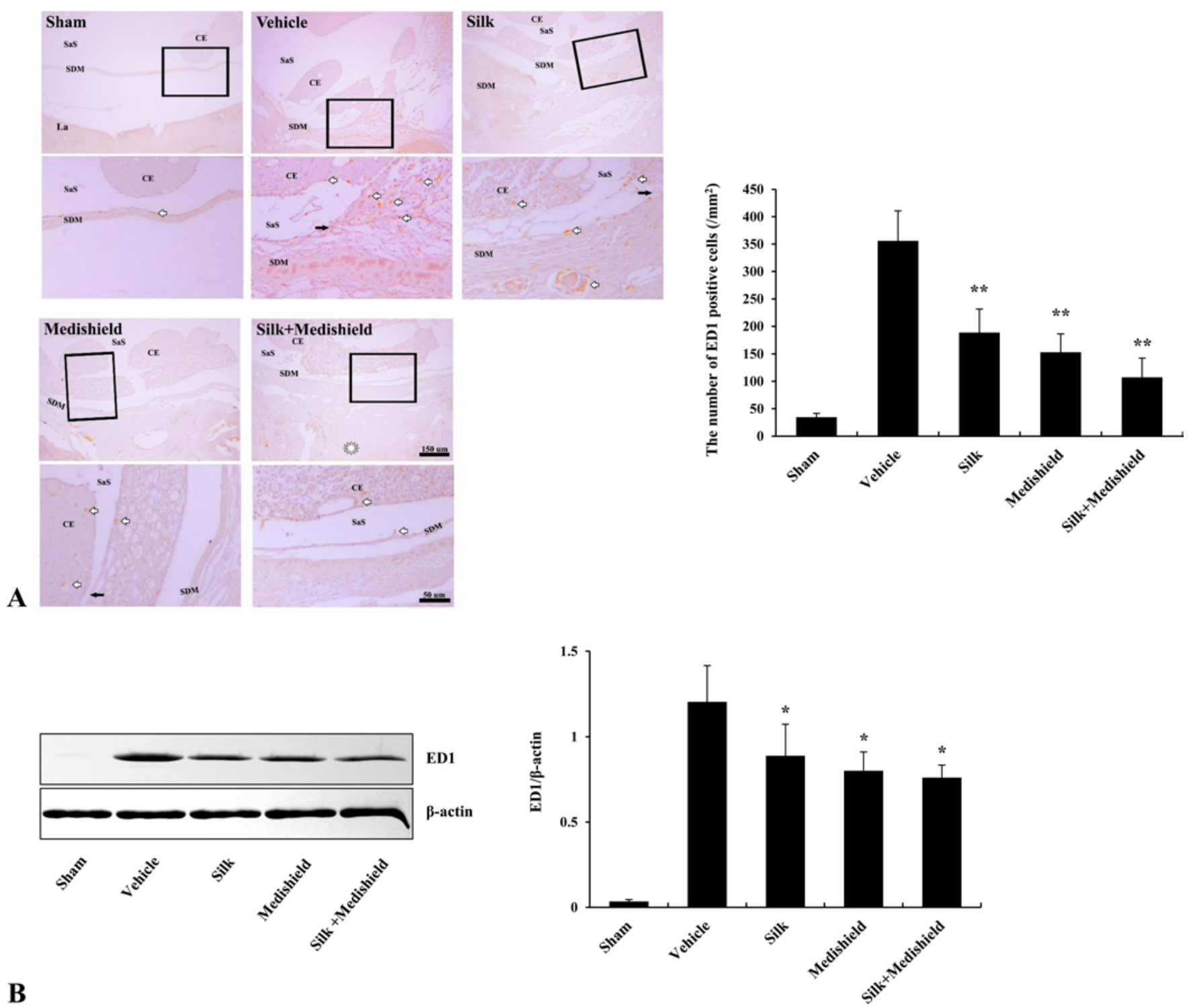

B

FIG. 3. Effects of silk solution on ED1 expression in rat dural adhesion model. Histological and Western blot analysis on silk solution treatment of dural adhesion. ED1 staining (A). Western blot analysis (B). High-magnification images are shown in bottom panels. Scale bar $=150 \mu \mathrm{m}$ (upper) and $50 \mu \mathrm{m}$ (lower). The difference between the vehicle group and the silk solution-treated group was statistically significant (graphs: ${ }^{*} p<0.05$ and ${ }^{* *} p<0.01$ ).

glial scar formation and inflammation after spinal cord injury. ${ }^{916}$ Esposito et al. showed that MMP-2 and MMP-9 are upregulated during spinal cord injury, whereas melatonin attenuates spinal cord injury in mice by inhibition of MMP-2 and MMP-9, suggesting that melatonin treatment may be useful in spinal cord injury. ${ }^{9}$

In agreement with these results, we showed that MMP-2 and MMP-9 expression levels were markedly increased in a laminectomy-induced adhesion rat animal model. However, silk solution treatment drastically reduced MMP-2 and MMP-9 expression levels compared to the laminectomy-induced adhesion rats.

ED1 is known as a marker of activated microglia, monocytes, and macrophages and can be useful to assess phagocytic activity in cells, which is considered to be a key factor in membrane ruffling by phagocytosis. ${ }^{26} \mathrm{CD} 34$, known as an adhesion marker protein, is highly expressed during inflammation and plays a role in cell adhesion and migration. Song et al. have shown that ED1 and CD34 protein expression levels were significantly increased in a rat adhesion model. ${ }^{33}$ Other studies have demonstrated that ED1 expression was markedly increased after spinal cord injury in a rat animal model. Therefore, these authors suggest that reduction of ED1 expression is an important factor to regulate the inflammatory cascade after spinal cord injury. ${ }^{1,34,41}$ In the present study, we showed that ED1 and CD34 expression levels were markedly reduced by silk solution in a laminectomy-induced adhesion rat model. 

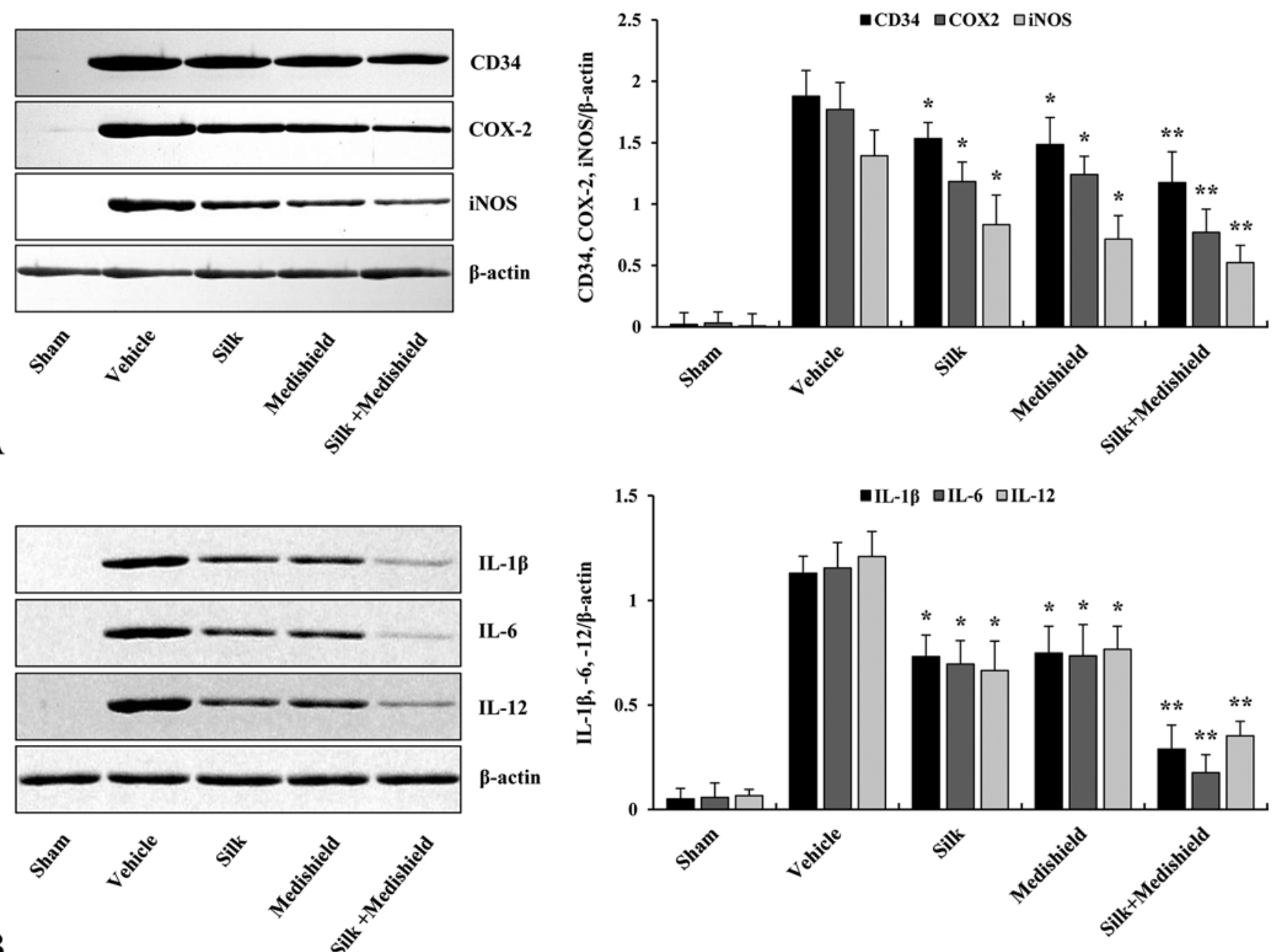

B

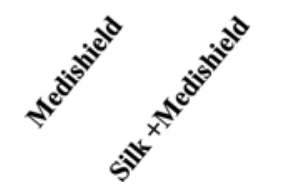

FIG. 4. Effects of silk solution on inflammatory response in rat dural adhesion model. The changes in inflammatory response expression levels were determined by Western blotting: changes of CD34, COX-2, and iNOS expression levels between the experimental groups (A) and changes in IL-1 $\beta$, IL-6, and IL-12 expression levels between the experimental groups (B). The difference between the vehicle group and the silk solution-treated group was statistically significant (graphs in A and $B:{ }^{*} p<0.05$ and ${ }^{* *} p<0.01$ ).

Inflammation causes the induction of COX-2, iNOS, and proinflammatory cytokines, including IL-1 $\beta$, IL-6, and IL-12.,28 Thus, inhibition of these products is one of the key strategies in an approach to the treatment of inflammation. Recent studies have shown that esculentoside A, a saponin, significantly inhibits lipopolysaccharide-induced proinflammatory cytokines and mediator proteins in BV2 and primary microglia cells. ${ }^{43}$ Also, other groups have shown that COX-2 inhibitors significantly inhibit intraabdominal adhesions in a murine adhesion model, suggesting that COX-2 inhibitors may prevent surgical adhesion formation in a murine model..$^{10}$ Here, we have shown that silk solution markedly inhibits laminectomy-induced expression of proinflammatory cytokines and mediator proteins. Taken together, we have demonstrated that silk solution significantly reduced dural adhesion formation and inflammation in a laminectomy-induced animal rat model. Although further studies are needed, including longer-term and larger-animal experiments, our results in- dicate that silk solution might be a promising therapeutic agent against dural adhesion formation.

\section{Conclusions}

Although dural adhesion formation is a major problem in surgical procedures, no effective treatment exists. Therefore, we prepared silk solution and examined its effect against laminectomy-induced dural adhesion formation and inflammation in a rat model. Silk solution significantly reduced postlaminectomy dural adhesion formation and inflammation. In addition, the effects of silk solution were similar to MediShield treatment. Although further research is necessary, our results suggest that silk solution may be a useful clinical material in neurosurgical procedures including laminectomy.

\section{Acknowledgments}

This study was supported by BioGreen 21 (PJ0131392018) of 
the Rural Development Administration, Republic of Korea. Additionally, this work was supported by a grant from Hallym University Medical Center Research Fund (HURF-2014-42).

\section{References}

1. Abdanipour A, Schluesener HJ, Tiraihi T: Effects of valproic acid, a histone deacetylase inhibitor, on improvement of locomotor function in rat spinal cord injury based on epigenetic science. Iran Biomed J 16:90-100, 2012

2. Block ML, Zecca L, Hong JS: Microglia-mediated neurotoxicity: uncovering the molecular mechanisms. Nat Rev Neurosci 8:57-69, 2007

3. Burton CV, Kirkaldy-Willis WH, Yong-Hing K, Heithoff KB: Causes of failure of surgery on the lumbar spine. Clin Orthop Relat Res (157):191-199, 1981

4. Caspar W, Campbell B, Barbier DD, Kretschmmer R, Gotfried Y: The Caspar microsurgical discectomy and comparison with a conventional standard lumbar disc procedure. Neurosurgery 28:78-87, 1991

5. Chen F, Zuo Z, Wang K, Zhang C, Gong H, Ye F, et al: Study on salvianolic acid B in the reduction of epidural fibrosis in laminectomy rats. BMC Musculoskelet Disord 15:337, 2014

6. Choi HJ, Kim KB, Kwon YM: Effect of amniotic membrane to reduce postlaminectomy epidural adhesion on a rat model. J Korean Neurosurg Soc 49:323-328, 2011

7. Cokluk C, Aydi K: Experimental rabbit hemilaminotomy model in the evaluation of peridural fibrosis: a minimally invasive peridural fibrosis model. Minim Invasive Neurosurg 48:235-239, 2005

8. Dumont RJ, Okonkwo DO, Verma S, Hurlbert RJ, Boulos PT, Ellegala DB, et al: Acute spinal cord injury, part I: pathophysiologic mechanisms. Clin Neuropharmacol 24:254264, 2001

9. Esposito E, Genovese T, Caminiti R, Bramanti P, Meli R, Cuzzocrea S: Melatonin regulates matrix metalloproteinases after traumatic experimental spinal cord injury. J Pineal Res 45:149-156, 2008

10. Greene AK, Alwayn IP, Nose V, Flynn E, Sampson D, Zurakowski D, et al: Prevention of intra-abdominal adhesions using the antiangiogenic COX-2 inhibitor celecoxib. Ann Surg 242:140-146, 2005

11. Hausmann ON: Post-traumatic inflammation following spinal cord injury. Spinal Cord 41:369-378, 2003

12. He Y, Revel M, Loty B: A quantitative model of postlaminectomy scar formation. Effects of a nonsteroidal antiinflammatory drug. Spine (Phila Pa 1976) 20:557-563, 579-580, 1995

13. Hellebrekers BWJ, Kooistra T: Pathogenesis of postoperative adhesion formation. Br J Surg 98:1503-1516, 2011

14. Henderson R, Weir B, Davis L, Mielke B, Grace M: Attempted experimental modification of the postlaminectomy membrane by local instillation of recombinant tissue-plasminogen activator gel. Spine (Phila Pa 1976) 18:1268-1272, 1993

15. Horan RL, Antle K, Collette AL, Wang Y, Huang J, Moreau $\mathrm{JE}$, et al: In vitro degradation of silk fibroin. Biomaterials 26:3385-3393, 2005

16. Hsu JY, Bourguignon LY, Adams CM, Peyrollier K, Zhang $\mathrm{H}$, Fandel T, et al: Matrix metalloproteinase-9 facilitates glial scar formation in the injured spinal cord. J Neurosci 28:13467-13477, 2008

17. Kim DW, Eum WS, Jang SH, Park J, Heo DH, Sheen SH, et al: A transparent artificial dura mater made of silk fibroin as an inhibitor of inflammation in craniotomized rats. J Neurosurg 114:485-490, 2011

18. Kim UJ, Park J, Li C, Jin HJ, Valluzzi R, Kaplan DL: Structure and properties of silk hydrogels. Biomacromolecules 5:786-792, 2004

19. Kitano T, Zerwekh JE, Edwards ML, Usui Y, Allen MD:
Viscous carboxymethylcellulose in the prevention of epidural scar formation. Spine (Phila Pa 1976) 16:820-823, 1991

20. Laurent GJ, Chambers RC, Hill MR, McAnulty RJ: Regulation of matrix turnover: fibroblasts, forces, factors and fibrosis. Biochem Soc Trans 35:647-651, 2007

21. Lower AM, Hawthorn RJ, Ellis H, O’Brien F, Buchan S, Crowe AM: The impact of adhesions on hospital readmissions over ten years after 8849 open gynaecological operations: an assessment from the Surgical and Clinical Adhesions Research Study. BJOG 107:855-862, 2000

22. Mauney JR, Nguyen T, Gillen K, Kirker-Head C, Gimble JM, Kaplan DL: Engineering adipose-like tissue in vitro and in vivo utilizing human bone marrow and adipose-derived mesenchymal stem cells with silk fibroin 3D scaffolds. Biomaterials 28:5280-5290, 2007

23. Meinel L, Hofmann S, Karageorgiou V, Kirker-Head C, McCool J, Gronowicz G, et al: The inflammatory responses to silk films in vitro and in vivo. Biomaterials 26:147-155, 2005

24. Mori H, Tsukada M: New silk protein: modification of silk protein by gene engineering for production of biomaterials. $\mathbf{J}$ Biotechnol 74:95-103, 2000

25. Mott JD, Werb Z: Regulation of matrix biology by matrix metalloproteinases. Curr Opin Cell Biol 16:558-564, 2004

26. Ohsawa K, Imai Y, Sasaki Y, Kohsaka S: Microglia/macrophage-specific protein Iba1 binds to fimbrin and enhances its actin-bundling activity. J Neurochem 88:844-856, 2004

27. Parker MC, Ellis H, Moran BJ, Thompson JN, Wilson MS, Menzies D, et al: Postoperative adhesions: ten-year follow-up of 12,584 patients undergoing lower abdominal surgery. Dis Colon Rectum 44:822-830, 2001

28. Ransohoff RM: How neuroinflammation contributes to neurodegeneration. Science 353:777-783, 2016

29. Robertson JT: Role of peridural fibrosis in the failed back: a review. Eur Spine J 5 (Suppl 1):S2-S6, 1996

30. Rockwood DN, Preda RC, Yücel T, Wang X, Lovett ML, Kaplan DL: Materials fabrication from Bombyx mori silk fibroin. Nat Protoc 6:1612-1631, 2011

31. Rodgers KE, Robertson JT, Espinoza T, Oppelt W, Cortese S, diZerega GS, et al: Reduction of epidural fibrosis in lumbar surgery with Oxiplex adhesion barriers of carboxymethylcellulose and polyethylene oxide. Spine J 3:277-284, 2003

32. Sandoval MA, Hernandez-Vaquero D: Preventing peridural fibrosis with nonsteroidal anti-inflammatory drugs. Eur Spine J 17:451-455, 2008

33. Song HJ, Kim JW, Park JS, Kim YS, Choi YS, Kim BG, et al: Effects of three different types of anti-adhesive agents in a rat abdominal wall defect model. J Korean Surg Soc 77:7-14, 2009

34. Stirling DP, Liu J, Plunet W, Steeves JD, Tetzlaff W: SB203580, a p38 mitogen-activated protein kinase inhibitor, fails to improve functional outcome following a moderate spinal cord injury in rat. Neuroscience 155:128-137, 2008

35. Sucu HK, Sevin IE, Rezanko T, Hamdi B, Sevin G: Prevention of anterior scar formation following discectomy with a MediShield adhesion barrier: randomized experimental trial. Turk Neurosurg 23:317-322, 2013

36. Tator $\mathrm{CH}$, Fehlings MG: Review of the secondary injury theory of acute spinal cord trauma with emphasis on vascular mechanisms. J Neurosurg 75:15-26, 1991

37. Turkoglu E, Dinc C, Tuncer C, Oktay M, Serbes G, Sekerci $\mathrm{Z}$ : Use of decorin to prevent epidural fibrosis in a post-laminectomy rat model. Eur J Pharmacol 724:86-91, 2014

38. Turkoglu E, Tuncer C, Dinc C, Serbes G, Oktay M, Sekerci $\mathrm{Z}$ : The effect of etanercept on spinal epidural fibrosis in a postlaminectomy rat model. Turk Neurosurg 24:506-511, 2014

39. Wang Y, Liang M, Zheng Z, Shi L, Su B, Liu J, et al: Adhe- 
sion prevention after laminectomy using silk-polyethylene glycol hydrogels. Adv Healthc Mater 4:2120-2127, 2015

40. Wang Z, Wang Y, Xie P, Liu W, Zhang S: Calcium channel blockers in reduction of epidural fibrosis and dural adhesions in laminectomy rats. Eur J Orthop Surg Traumatol 24 (1 Suppl 1):S293-S298, 2014

41. Wiens R, Rak M, Cox N, Abraham S, Juurlink BH, Kulyk WM, et al: Synchrotron FTIR microspectroscopic analysis of the effects of anti-inflammatory therapeutics on wound healing in laminectomized rats. Anal Bioanal Chem 387:16791689, 2007

42. Yan C, Boyd DD: Regulation of matrix metalloproteinase gene expression. J Cell Physiol 211:19-26, 2007

43. Yang H, Chen Y, Yu L, Xu Y: Esculentoside A exerts antiinflammatory activity in microglial cells. Int Immunopharmacol 51:148-157, 2017

44. Zhang C, Kong X, Ning G, Liang Z, Qu T, Chen F, et al: Alltrans retinoic acid prevents epidural fibrosis through NF- $\mathrm{KB}$ signaling pathway in post-laminectomy rats. Neuropharmacology 79:275-281, 2014

45. Zhu J, Li Y, Shen W, Qiao C, Ambrosio F, Lavasani M, et al: Relationships between transforming growth factor-beta1, myostatin, and decorin: implications for skeletal muscle fibrosis. J Biol Chem 282:25852-25863, 2007

\section{Disclosures}

The authors report no conflict of interest concerning the materials or methods used in this study or the findings specified in this paper.

\section{Author Contributions}

Conception and design: Choi, Cho, CH Lee, Kim, Yoo. Acquisition of data: Eum, Shin, Jo. Analysis and interpretation of data: Park, Han, KW Lee. Critically revising the article: Choi.

\section{Correspondence}

Soo Young Choi: Hallym University, Chuncheon, Korea. sychoi@ hallym.ac.kr. 